\title{
Persistência e liberação de elementos da fitomassa do consórcio crotalária com milheto sob fragmentação ${ }^{1}$
}

\author{
Persistence and release of elements in the biomass from sunn hemp intercropped with \\ millet under fragmentation
}

\author{
Claudio Hideo Martins da Costa ${ }^{2}$, Carlos Alexandre Costa Crusciol ${ }^{3 *}$, Rogério Peres Soratto ${ }^{4}$ e Jayme Ferrari \\ Neto $^{2}$
}

\begin{abstract}
RESUMO - Objetivou-se, com esse trabalho, avaliar a taxa de decomposição e a velocidade de liberação de macronutrientes e Si da fitomassa do consórcio crotalária mais milheto, em função do tempo após manejo, sem e com fragmentação. O experimento foi conduzido em condições de campo, em Botucatu-SP. O delineamento experimental foi de blocos casualizados, com quatro repetições, em esquema fatorial constituído por dois manejos da fitomassa da parte aérea (sem e com fragmentação mecânica) e seis épocas de coleta $[0 ; 18 ; 32 ; 46 ; 74$ e 91 dias após manejo (DAM)]. Foram determinadas a massa de matéria seca, teor de macronutrientes e Si e quantidade dos nutrientes remanescentes. Os dados foram submetidos à análise de variância, as médias dos tratamentos do fator manejo comparadas pelo teste de t(LSD) a 5\% e do fator épocas de coletas ajustados a equações matemáticas. A fragmentação da fitomassa aumenta a taxa de decomposição e a liberação de N, P, Ca e S. O K é rapidamente disponibilizado, restando 91 DAM, em média, 1,5\% da quantidade total acumulada. Aos 91 DAM pelo menos $80 \%$ de todos os macronutrientes já haviam sido liberados ao solo. A máxima liberação diária dos macronutrientes ocorre entre 0 a $18 \mathrm{DAM}$. A taxa de liberação de $\mathrm{Si}$ é constante e ocorre aumento do teor do elemento na fitomassa com o tempo devido à acentuada liberação de C. O Si é o elemento liberado mais lentamente ao solo, restando ainda 91 DAM, em média, $85 \%$ da quantidade total acumulada na fitomassa.
\end{abstract}

Palavras-chave: Crotalaria juncea. Milheto. Palha-utilização na agricultura. Cultivo consorciado.

\begin{abstract}
The objective of this work was to evaluate the rate of decomposition and rate of release of macronutrients and $\mathrm{Si}$ in the biomass from intercropped sunn hemp and millet, as a function of time after management, both with and without fragmentation. The experiment was conducted under field conditions in Botucatu, São Paulo. The experimental design was of randomized blocks with four replications, in a factorial design consisting of two managements of shoot phytomass (with and without mechanical fragmentation) at six harvesting times [0, 18, 32, 46, 74 and 91 days after management (DAM)]. The dry-matter weight, macronutrient and Si content, and quantity of remaining nutrients were determined. The data were subjected to variance analysis, the averages of the factor management treatments were compared by t-test (LSD) at 5\%, and those of the factor sampling-times, adjusted to the mathematical equations. Fragmentation of the phytomass increases the rate of decomposition and release of $\mathrm{N}, \mathrm{P}, \mathrm{Ca}$ and $\mathrm{S} . \mathrm{K}$ is available quickly, with on average $1.5 \%$ of the total amount accumulated at 91 DAM. By 91 DAM at least $80 \%$ of all the macronutrients had been released into the soil. The maximum daily release of macronutrients occurs between 0 to18 DAM. The release rate of $\mathrm{Si}$ is constant, and there is an increase of the content of this element in the phytomass over time, due to the marked release of $\mathrm{C}$. The element $\mathrm{Si}$ is that released most slowly into the soil, with there still being present at DAM 91 on average $85 \%$ of the total amount accumulated in the phytomass.
\end{abstract}

Key words: Crotalaria juncea. Pearl millet. Straw-utilization in agriculture. Intercropping.

\footnotetext{
*Autor para correspondência

'Recebido para publicação em 07/02/2011; aprovado em 08/09/2013

Pesquisa realizada com recursos financeiros da FAPESP

${ }^{2}$ Faculdade de Ciências Agronômicas, Universidade Estadual Paulista/UNESP, Caixa Postal 237, Botucatu-SP, Brasil, 18.610-307, chmcosta@ fca.unesp.br, jfneto@fca.unesp.br

${ }^{3}$ Departamento de Produção e Melhoramento Vegetal da FCA/UNESP, José Barbosa de Barros, 1780, Caixa Postal 237, Botucatu-SP, Brasil, 18.610-307, crusciol@fca.unesp.br

${ }^{4}$ Departamento de Produção e Melhoramento Vegetal da FCA/UNESP, soratto@ fca.unesp.br
} 


\section{INTRODUÇÃO}

No sistema plantio direto (SPD), a manutenção da palhada sobre a superfície do solo promove maior proteção contra o impacto direto das gotas de chuva, favorece a infiltração, reduz a perda de água por escoamento superficial e a perda de solo e nutrientes por erosão (CASTRO et al., 1986; HERNANI; KURIHARA; SILVA, 1999). Contudo, especialmente nas regiões Sudeste e Centro-Oeste do Brasil, a produção e a manutenção de cobertura vegetal na superfície do solo é um desafio, devido ao inverno seco, que limita o cultivo nessa época, e o verão quente e chuvoso, que aceleram a decomposição da palhada (BOER et al., 2008; LEITE et al., 2010; SILVA et al., 2010). Assim, a quantidade e a durabilidade da fitomassa, bem como sua capacidade de reciclar nutriente, são características importantes a serem consideradas na escolha das plantas de cobertura (BOER et al., 2008; LEITE et al., 2010).

Entre as espécies utilizadas como plantas de cobertura e para adubação verde, duas se destacam: a crotalária (Crotalaria juncea L.), pela boa cobertura do solo proporcionado pelo rápido crescimento, pela associação simbiótica com bactérias fixadoras de $\mathrm{N}_{2}$ (PERIN; GUERRA; TEIXEIRA, 2003) e supressão de nematóides parasitas (INOMOTO et al., 2008), sendo que essa última característica tem acarretado em grande expansão do cultivo dessa espécie nas áreas de produção de grãos na região do cerrado brasileiro; e o milheto (Pennisetum glaucum (L.) R. Brown), que é caracterizado pela produção de palhada em grande quantidade e com características de maior persistência sobre o solo (BOER et al., 2008; SILVA et al., 2010), pela alta capacidade de extração de nutrientes do solo, com amplas vantagens de reciclagem, principalmente $\mathrm{N}$ e K, reduzindo os riscos de lixiviação (ROSOLEM; PACE; CRUSCIOL, 2004; CRUSCIOL; SORATTO, 2007, 2009; LEITE et al., 2010).

O cultivo consorciado, com plantas de cobertura entre gramíneas e leguminosas, pode ser uma alternativa viável para o sistema de rotação quanto ao fornecimento de maior quantidade e qualidade de fitomassa (AITA; GIACOMINI, 2003; PERIN et al., 2004, 2006). O consórcio crotalária mais milheto, além de fornecer palhada com boa persistência (CAZZETA et al., 2005), incrementa principalmente os teores de $\mathrm{K}$ e $\mathrm{N}$ no solo, elementos importantes para as culturas em sucessão (PERIN et al., 2004, 2006).

No SPD, poucas foram as pesquisas que visaram estudar o tipo de manejo da fitomassa e sua relação com os processos de liberação de nutrientes e decomposição da mesma (REIS et al., 2007). Porém, é possível que o tipo de manejo influencie diretamente a velocidade de liberação de nutrientes, bem como, a degradação da cobertura morta na superfície do solo. Assim, é de fundamental importância o conhecimento da dinâmica de liberação de nutrientes da cobertura vegetal em função do manejo utilizado no material vegetal, para que se possa compatibilizar esse aspecto com as exigências da cultura sucedânea (BOER et al., 2007; LEITE et al., 2010).

Desta forma, objetivou-se avaliar persistência da fitomassa do consórcio crotalária com milheto, bem como, a velocidade de liberação dos macronutrientes e $\mathrm{Si}$ e as mudanças nas relações $\mathrm{C} / \mathrm{N}, \mathrm{C} / \mathrm{P}, \mathrm{C} / \mathrm{S}$ e C/Si na fitomassa, em função da fragmentação.

\section{MATERIAL E MÉTODOS}

O trabalho foi desenvolvido em condições de campo, na Fazenda Experimental Lageado da Faculdade de Ciências Agronômicas da UNESP, em Botucatu (SP) ( $22^{\circ} 58^{\prime} \mathrm{S}, 48^{\circ} 23^{\prime} \mathrm{W}$ e $765 \mathrm{~m}$ de altitude). O solo do local é um Nitossolo Vermelho. Segundo a classificação climática de Köeppen, o clima predominante na região é do tipo Cwa, caracterizado como clima tropical de altitude, com inverno seco e verão quente e chuvoso.

Durante a condução do experimento a precipitação pluviométrica e a temperatura média mensal foram de, respectivamente, $430 \mathrm{~mm}$ e $23{ }^{\circ} \mathrm{C}$ em janeiro, $70 \mathrm{~mm}$ e $23,5^{\circ} \mathrm{C}$ em fevereiro, $120 \mathrm{~mm}$ e $23,5^{\circ} \mathrm{C}$ em março e $88 \mathrm{~mm}$ e $22,8^{\circ} \mathrm{C}$ em abril.

As características químicas do solo $(0-20 \mathrm{~cm})$ foram determinadas antes da instalação do experimento e os resultados foram: $25,0 \mathrm{~g} \mathrm{dm}^{-3}$ de matéria orgânica, 5,0 de $\mathrm{pH}\left(\mathrm{CaCl}_{2}\right), 17 \mathrm{mg} \mathrm{dm}^{-3}$ de $\mathrm{P}$ (resina), 1,6; 33,3; 17,6 e $34,1 \mathrm{mmol}_{\mathrm{c}} \mathrm{dm}^{-3} \mathrm{de} \mathrm{K}, \mathrm{Ca}, \mathrm{Mg}$ e $\mathrm{H}+\mathrm{Al}$, respectivamente, e $61 \%$ de saturação por bases.

$\mathrm{O}$ delineamento experimental foi em blocos casualizados, disposto em esquema fatorial, constituídos por dois manejos da fitomassa (sem e com manejo fragmentação) e seis épocas de coleta da fitomassa da parte aérea $(0 ; 18 ; 32 ; 46 ; 74$ e 91 dias após o manejo (DAM)), com quatro repetições. As dimensões das parcelas foram de $5 \mathrm{~m}$ de largura e $15 \mathrm{~m}$ de comprimento, perfazendo $75 \mathrm{~m}^{2}$.

Antes da semeadura das espécies de pantas de cobertura, foi realizada a dessecação das plantas presentes na área com a utilização do herbicida glyphosate na dose de $4 \mathrm{~L} \mathrm{ha}^{-1}$ do produto comercial (1.920 $\mathrm{g} \mathrm{ha}^{-1}$ i.a.). A semeadura do consórcio crotalária (cv. IAC-KR1) com milheto (cv. BN-2), foi efetuada em 21/10/2004, cuja emergência ocorreu nove dias após (30/10/2004). Utilizou-se a mistura de $20 \mathrm{~kg} \mathrm{ha}^{-1}$ de sementes crotalária 
com $10 \mathrm{~kg} \mathrm{ha}^{-1}$ de milheto, no espaçamento de $0,17 \mathrm{~m}$ entre linhas, e aproximadamente $0,03 \mathrm{~m}$ de profundidade. Não foi realizada qualquer adubação, assim como tratos fitossanitários.

Aos 75 dias após a emergência(DAE), em 14/01/2005, foi realizado o manejo químico com a utilização do herbicida glyphosate na dose de $4 \mathrm{~L} \mathrm{ha}^{-1}$ do produto comercial (1.920 $\mathrm{g} \mathrm{ha}^{-1}$ i.a.). Após a dessecação, realizou-se o manejo mecânico por meio de triturador de palha horizontal, nas parcelas previamente determinadas.

Realizou-se coletas da cobertura vegetal no dia do manejo (0 DAM), em 01/02/2005 (18 DAM), em 15/02/2005 (32 DAM), em 01/03/2005 (46 DAM), em 29/03/2005 (74 DAM) e em 15/04/2005 (91 DAM). Em cada época de coleta foram amostrados três quadros, conforme a metodologia proposta por Crusciol et al. (2005), com $0,25 \mathrm{~m}^{2}$ de área interna (amostras simples), que constituíram uma amostra composta por parcela. O caminhamento de amostragem, nas unidades experimentais, foi realizado no sentido diagonal, sendo aleatória a escolha dos pontos de coleta, excluindo-se $0,50 \mathrm{~m}$ de cada extremidade como bordadura.

Os resíduos sofreram pré-limpeza por meio de peneiras, para remoção do solo aderido, em seguida foram lavados, seguindo metodologia de Malavolta, Vitti e Oliveira (1997), porém modificada, ou seja, sem o emprego de detergente, conforme Rosolem, Calonego e Foloni (2003). Assim, as amostras foram agitadas por alguns segundos em água deionizada, em três porções sucessivas, sendo depois colocados sobre papel absorvente. Ressalta-se que a não utilização de detergente diminui o tempo de exposição à agitação com água e o número de porções sucessivas, o que reduziu ao máximo as prováveis perdas de $\mathrm{K}$ da fitomassa (ROSOLEM; CALONEGO; FOLONI, 2003). As amostras foram acondicionadas em sacos de papel e secas em estufa com circulação forçada de ar a $60{ }^{\circ} \mathrm{C}$, e pesadas, para determinação da massa de matéria seca. A seguir, o material foi moído em moinho tipo Willey, para posterior determinação dos teores de macronutrientes (MALAVOLTA; VITTI; OLIVEIRA, 1997), carbono (TEDESCO; WOLKWEISS; BOHNEN, 1985) e silício (KORNDÖRFER; PEREIRA; CAMARGO, 2002).

As quantidades de macronutrientes, $\mathrm{C}$ e $\mathrm{Si}$ remanescentes na fitomassa foram determinadas pelo produto da quantidade de massa seca e os teores dos elementos do resíduo vegetal em cada época de coleta. De posse desses valores, calculou-se a degradação da fitomassa e a quantidade dos elementos contida na palhada, sendo os dados apresentados em $\mathrm{kg} \mathrm{ha}^{-1}$. Esse resultado também foi expresso em porcentagem (\%) pela relação: quantidade remanescente de palhada ou de cada nutriente, em cada época, pela quantidade inicial, multiplicado por 100.
Para descrever a decomposição da fitomassa e a quantidade remanescente dos elementos $(\mathrm{N}, \mathrm{P}, \mathrm{K}, \mathrm{Ca}$, $\mathrm{Mg}, \mathrm{S}$, e C) na mesma, tanto em $\mathrm{kg} \mathrm{ha}^{-1}$ quanto em $\%$, foi utilizado o modelo matemático exponencial descrito por Thomas e Asakawa (1993), conforme equação 1:

$\mathrm{X}=\mathrm{Xoe}^{-\mathrm{kt}}$

em que $X$ é a quantidade de fitomassa seca ou de elementos remanescente após um período de tempo $t$, em dias; Xo é a quantidade inicial de fitomassa seca ou de elementos; e k é a constante de decomposição do resíduo ou liberação dos elementos.

Com o valor de $\mathrm{k}$, foi calculado o tempo de meia-vida $\left(\mathrm{t}^{\mathrm{1}} \mathrm{2}=0,693 / \mathrm{k}\right)$ (PAUL; CLARK, 1989), que expressa o período de tempo necessário para que metade dos resíduos se decomponha ou para que metade dos elementos contidos nos resíduos seja liberada. Aplicandose a derivada primeira às funções ajustadas aos dados de fitomassa e liberação acumulada dos elementos, calculouse as taxas diárias de decomposição de fitomassa e de liberação dos elementos após o manejo das plantas de cobertura (KLIEMANN; BRAZ; SILVEIRA, 2006; ROSOLEM; CALONEGO; FOLONI, 2003).

Os dados foram submetidos à análise de variância. As médias dos tratamentos do fator manejo da fitomassa foram comparadas pelo teste de $\mathrm{t}$ (LSD) a 5\% de probabilidade, e os dados do fator épocas de coletas da fitomassa foram ajustados a equações matemáticas, adotando-se como critério para escolha do modelo, a magnitude dos coeficientes de regressão significativos a 5\% de probabilidade pelo teste F. Foi utilizado o programa estatístico Sisvar.

\section{RESULTADOS E DISCUSSÃO}

Os dados do fator épocas de coletas da fitomassa foram ajustados a equações matemáticas dentro de cada manejo (Tabela 1).

A produção de fitomassa seca da parte aérea do consórcio, aos 75 DAE, foi de aproximadamente $8.470 \mathrm{~kg} \mathrm{ha}^{-1}$ (Figura 1A), valor próximo aos obtidos por Perin et al. (2004) e Menezes et al. (2009), que foram de $8.040 \mathrm{~kg} \mathrm{ha}^{-1}$, aos $60 \mathrm{DAE}$, e $9.183 \mathrm{~kg} \mathrm{ha}^{-1}$, aos 90 DAE, respectivamente.

A decomposição da fitomassa do consórcio foi ajustada a equações exponenciais, tanto sem quanto com fragmentação mecânica (Tabela 1). A decomposição atingiu 50\% da quantidade inicial aos 57 e 48 DAM, sem e com fragmentação da fitomassa, respectivamente, sendo os valores ajustados a funções exponenciais, semelhante nos dois tipos de manejo (Figuras 1A e 1B). 
Tabela 1 - Análise de variância das equações matemáticas em função tempo após manejo em cada um dos manejos da fitomassa das variáveis relacionadas à matéria seca, relação entre carbono e nutrientes e quantidade remanescente de nutrientes na fitomassa do consórcio crotalária (Crotalaria juncea) + milheto (Pennisetum glaucum)

\begin{tabular}{|c|c|c|c|c|}
\hline \multirow{3}{*}{ Variável } & \multicolumn{4}{|c|}{ Manejo da fitomassa } \\
\hline & \multicolumn{2}{|c|}{ Sem fragmentação } & \multicolumn{2}{|c|}{ Com fragmentação } \\
\hline & Regressão & Valor de $\mathrm{F}$ & Regressão & Valor de F \\
\hline Matéria seca $\left(\mathrm{kg} \mathrm{ha}^{-1}\right)$ & Exponencial & $248,32 * *$ & Exponencial & $430,93 * *$ \\
\hline Matéria seca (\%) & Exponencial & $243,66 * *$ & Exponencial & $387,17^{* *}$ \\
\hline Relação C/N & Quadrática & $27,74 * *$ & Linear & $89,63^{* *}$ \\
\hline Relação C/P & Quadrática & $21,14 * *$ & Linear & $112,85^{* *}$ \\
\hline Relação C/S & Linear & $142,79 * *$ & Linear & $404,96 * *$ \\
\hline Relação C/Si & Quadrática & $20,11 * *$ & Quadrática & $37,33^{* *}$ \\
\hline Teor de $\mathrm{N}\left(\mathrm{g} \mathrm{kg}^{-1}\right)$ & Quadrática & $48,64 * *$ & Quadrática & $30,69 * *$ \\
\hline Teor de $\mathrm{P}\left(\mathrm{g} \mathrm{kg}^{-1}\right)$ & Quadrática & $29,90 * *$ & Quadrática & $13,62 * *$ \\
\hline Teor de $\mathrm{K}\left(\mathrm{g} \mathrm{kg}^{-1}\right)$ & Quadrática & $558,67 * *$ & Quadrática & $711,61 * *$ \\
\hline Teor de Ca $\left(\mathrm{g} \mathrm{kg}^{-1}\right)$ & Quadrática & $13,52 * *$ & Quadrática & $35,95^{* *}$ \\
\hline Teor de $\mathrm{Mg}\left(\mathrm{g} \mathrm{kg}^{-1}\right)$ & Linear & $50,74 * *$ & Linear & $102,00 * *$ \\
\hline Teor de S $\left(\mathrm{g} \mathrm{kg}^{-1}\right)$ & Quadrática & $12,71^{* *}$ & Quadrática & $29,49^{* *}$ \\
\hline Teor de $\mathrm{C}\left(\mathrm{g} \mathrm{kg}^{-1}\right)$ & Linear & $3,97 * *$ & Linear & $60,48^{* *}$ \\
\hline Teor de Si $\left(\mathrm{g} \mathrm{kg}^{-1}\right)$ & Linear & $130,21 * *$ & Linear & $210,62^{* *}$ \\
\hline Quantidade de $N\left(\mathrm{~kg} \mathrm{ha}^{-1}\right)$ & Exponencial & $182,23 * *$ & Exponencial & $410,66 * *$ \\
\hline Quantidade de $\mathrm{P}\left(\mathrm{kg} \mathrm{ha}^{-1}\right)$ & Exponencial & $265,49 * *$ & Exponencial & $712,24 * *$ \\
\hline Quantidade de K $\left(\mathrm{kg} \mathrm{ha}^{-1}\right)$ & Exponencial & $697,58 * *$ & Exponencial & $3262,52 * *$ \\
\hline Quantidade de Ca $\left(\mathrm{kg} \mathrm{ha}^{-1}\right)$ & Exponencial & $324,55^{* *}$ & Exponencial & $575,21 * *$ \\
\hline Quantidade de $\operatorname{Mg}\left(\mathrm{kg} \mathrm{h}^{-1}\right)$ & Exponencial & $258,97 * *$ & Exponencial & $204,83^{* *}$ \\
\hline Quantidade de S $\left(\mathrm{kg} \mathrm{ha}^{-1}\right)$ & Exponencial & $1096,53^{* *}$ & Exponencial & $1202,38 * *$ \\
\hline Quantidade de C $\left(\mathrm{kg} \mathrm{ha}^{-1}\right)$ & Exponencial & $423,28 * *$ & Exponencial & $438,99 * *$ \\
\hline Quantidade de Si $\left(\mathrm{kg} \mathrm{ha}^{-1}\right)$ & Linear & $2,23 * *$ & Linear & $3,46^{* *}$ \\
\hline $\mathrm{N}$ remanescente $(\%)$ & Exponencial & $198,57 * *$ & Exponencial & $454,94 * *$ \\
\hline P remanescente (\%) & Exponencial & $246,61 * *$ & Exponencial & $716,49 * *$ \\
\hline $\mathrm{K}$ remanescente $(\%)$ & Exponencial & $917,95 * *$ & Exponencial & $5475,51 * *$ \\
\hline Ca remanescente $(\%)$ & Exponencial & $410,95 * *$ & Exponencial & $575,01 * *$ \\
\hline $\mathrm{Mg}$ remanescente $(\%)$ & Exponencial & $289,35^{* *}$ & Exponencial & $206,13 * *$ \\
\hline S remanescente $(\%)$ & Exponencial & $1281,56^{* *}$ & Exponencial & $1453,89 * *$ \\
\hline C remanescente $(\%)$ & Exponencial & $498,39 * *$ & Exponencial & $488,58 * *$ \\
\hline Si remanescente $(\%)$ & Linear & $1,79 * *$ & Linear & $3,09 * *$ \\
\hline
\end{tabular}

**é significativo a $1 \%$ de probabilidade pelo teste $\mathrm{F}$

Na última amostragem (91 DAM) restavam apenas, 34 e $28 \%$ da quantidade de fitomassa inicial, sem e com fragmentação da fitomassa, respectivamente. Perin et al. (2006), com o corte e disposição da palhada do consórcio sobre o solo, observaram que o tempo necessário para decomposição de $50 \%$ da fitomassa foi de 41 dias. Esse menor período de decomposição constatado pelos autores pode estar relacionado à maior precipitação pluvial $(910 \mathrm{~mm})$ ocorrida em relação ao presente trabalho $(708 \mathrm{~mm})$, que proporcionou maior umidade, um dos fatores que influencia diretamente o processo de decomposição (MOREIRA; SIQUEIRA, 
Figura 1 - Matéria seca $\left(\mathrm{kg} \mathrm{ha}^{-1}\right)(\mathrm{A})$, porcentagem remanescente de matéria seca (\%) (B), taxa diária de decomposição da matéria seca $\left(\mathrm{kg} \mathrm{ha}^{-1} \mathrm{dia}^{-1}\right)(\mathrm{C})$, relações C/N (D), C/P (E), C/S (F) e C/Si (G) da fitomassa do consórcio crotalária (Crotalaria juncea) + milheto (Pennisetum glaucum) em função do tempo após o manejo (Dias após manejo), sem $(-\bullet)$ e com (- $-\cdots)$ fragmentação mecânica. Botucatu, SP. Barras verticais são indicativas do valor de DMS a 5\% de probabilidade
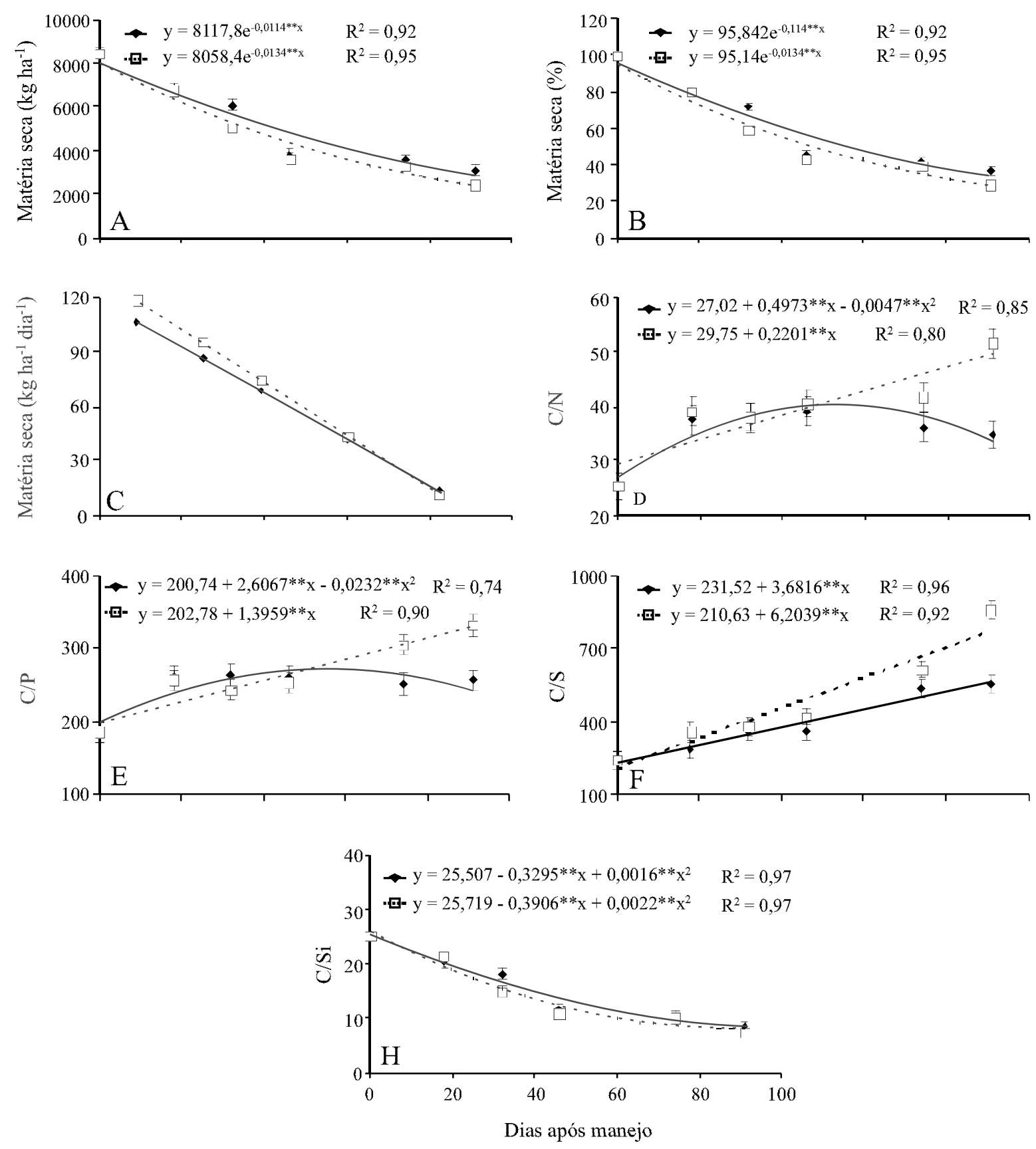

2006), ou pelo fato das plantas de cobertura terem sido manejada mais precocemente (68 dias após a semeadura) que no presente trabalho.

Essa constatação é embasada pelos resultados das relações $\mathrm{C} / \mathrm{N}, \mathrm{C} / \mathrm{P}, \mathrm{C} / \mathrm{S}$ e $\mathrm{C} / \mathrm{Si}$, que expressam a resistência da fitomassa à decomposição. No momento do manejo os valores das relações $\mathrm{C} / \mathrm{N}, \mathrm{C} / \mathrm{P}, \mathrm{C} / \mathrm{S}$ e $\mathrm{C} / \mathrm{Si}$ eram, respectivamente de $25 ; 187 ; 245$ e 25 (Figuras 1D, 1E, 1F e 1H). No entanto, com o passar do tempo as relações $\mathrm{C} / \mathrm{N}, \mathrm{C} / \mathrm{P}$ e $\mathrm{C} / \mathrm{S}$ aumentaram e a de $\mathrm{C} / \mathrm{Si}$ diminuiu, atingindo na última amostragem, 
respectivamente, valores de $34 ; 246 ; 567$ e 8 , sem a fragmentação da fitomassa, e de 50; 329; 775 e 8 com a fragmentação. A fragmentação da fitomassa acelerou o processo de liberação dos macronutrientes $\mathrm{N}$, P e S (Figuras 2A, e 2B), pois os valores das relações foram maiores (Figuras 1D, 1E e 1F). Como esses macronutrientes estão associados a componentes orgânicos do tecido vegetal (MARSCHNER, 1995), eles são facilmente liberados da fitomassa e com maior intensidade quando há fragmentação.

A fitomassa restante na última coleta (91 DAM) tinha grande concentração de $\mathrm{Si}$ na sua constituição (Figura 2H), sendo um tecido altamente silicificado, portanto de difícil degradação, denominado de fitólitos. $\mathrm{O} \mathrm{Si}$ ao ser absorvido pela planta polimeriza-se na forma de fitólitos, sílica amorfa de difícil decomposição, que se acumulam principalmente no entorno ou dentro das células do tecido vegetal (PINILLA, 1997). Assim, conforme a fitomassa é degradada, aumenta a concentração dos fitólitos no resíduo vegetal, até que a alta concentração diminui a taxa de decomposição (Figura 1C). Apesar do aumento da concentração de fitólitos, decorrente do aumento da relação $\mathrm{C} / \mathrm{Si}$, o mesmo não resultou em menor taxa de decomposição da fitomassa. Dessa forma, a relação $\mathrm{C} / \mathrm{Si}$ precisa ser mais estudada para estabelecer a sua devida importância como variável para auxiliar na explicação da dinâmica de decomposição das palhadas das diversas espécies utilizadas como plantas de cobertura do solo.

Entretanto, em relação a outras espécies de cobertura, como o nabo forrageiro (CRUSCIOL et al., 2005) e a aveia preta (CRUSCIOL et al., 2008), a fitomassa do consórcio tem boa persistência, independentemente do manejo utilizado, evidenciando que, nas condições experimentais, a fragmentação refletiu em um ligeiro aumento na degradação da palhada.

Mediante a Figura 2, verifica-se que houve redução gradual dos teores de todos os macronutrientes e aumento do teor de Si na fitomassa remanescente. Esse efeito ocorreu à medida que diminuiu a quantidade da fitomassa sobre o solo (Figura 1A). Assim, comparando os teores iniciais com os atingidos na última avaliação, verificou-se reduções de 47, 56, 89, 50, 60, 80 e $13 \%$ com fragmentação, e de $34 ; 31 ; 94 ; 36 ; 43 ; 65$ e $21 \%$, sem fragmentação da fitomassa, respectivamente, para $\mathrm{N}, \mathrm{P}, \mathrm{K}, \mathrm{Ca}, \mathrm{Mg}, \mathrm{S}$ e C.

Especificamente, quanto ao teor $\mathrm{K}$, constatou-se acentuada liberação inicial deste elemento, com posterior redução, provavelmente em função da baixa quantidade de $\mathrm{K}$ remanescente no tecido vegetal (Figuras 2C e 3C). Resultados semelhantes foram observados por Crusciol et al. (2005, 2008), Boer et al. (2007) e Leite et al. (2010). A rápida liberação de $\mathrm{K}$ da fitomassa ocorreu em decorrência desse elemento não estar associado a nenhum componente estrutural do tecido vegetal (MARSCHNER, 1995). O K não é metabolizado na planta, formando ligações com complexos orgânicos de fácil reversibilidade (ROSOLEM; CANOLEGO; FOLONI, 2003). Assim, à medida que a parte aérea das plantas do consórcio inicia o processo de secagem e se degrada, a concentração desse nutriente no tecido diminui drasticamente, pois é facilmente lavado pela água das chuvas (KHATOUNIAN, 1999), após o rompimento das membranas plasmáticas (MALAVOLTA; VITTI; OLIVEIRA, 1997).

Com relação ao $\mathrm{Si}$, o aumento dos teores na fitomassa no decorrer das amostragens, 150 e $200 \%$ aos 91 DAM, respectivamente, sem e com fragmentação mecânica, em relação aos valores iniciais (Figura $2 \mathrm{H}$ ), foi, provavelmente, em razão do aumento da concentração de fitólitos à medida que a fitomassa foi se degradando.

Quanto ao manejo da fitomassa, observou-se maior decréscimo nos teores de $\mathrm{N}, \mathrm{P}, \mathrm{Ca}, \mathrm{Mg}$ e $\mathrm{S}$ quando a mesma foi fragmentada (Figuras 2A, 2B, 2D, 2E e 2F). Esses resultados podem ser atribuídos ao maior contato da fitomassa com o solo, liberando mais facilmente esses nutrientes que estão associados a componentes orgânicos do tecido vegetal (MARSCHNER, 1995).

No momento do manejo, o acúmulo aproximado de $\mathrm{N}, \mathrm{P}$ e $\mathrm{K}$ foi, respectivamente, 161, 22 e $136 \mathrm{~kg} \mathrm{ha}^{-1}$ (Figuras 3A, 3B e 3C). Comparando aos resultados obtidos por Cazetta, Fornasieri Filho e Girotto (2005), esses valores expressam quantidades semelhantes de N, P e K reciclados pelo consórcio crotalária+milheto. Quantidades expressivas também foram constatadas quanto ao acúmulo de $\mathrm{Ca}, \mathrm{Mg}, \mathrm{S}, \mathrm{C}$ e Si, que atingiram valores aproximados da ordem de 46, 22, 17, 4.041 e $162 \mathrm{~kg} \mathrm{ha}^{-1}$, respectivamente (Figuras 3D, 3E, 3F, 3G e 3H).

A quantidade remanescente do nutriente na palhada é indicativo da quantidade disponibilizada para o solo. Assim, restaram na última avaliação 29,0; 4,1; 2,$2 ; 7,9 ; 4,5 ; 1,9 ; 1.109$ e $141,2 \mathrm{~kg} \mathrm{ha}^{-1}$, nas parcelas sem a fragmentação da fitomassa, e 19,5; 2,9; 1,8; 5,$4 ; 2,8 ; 1,2,981$ e $134,4 \mathrm{~kg} \mathrm{ha}^{-1}$ com fragmentação mecânica, respectivamente, de N, P, K, Ca, Mg, S, C e Si (Figura 3). Dessa forma, na última avaliação tinha sido disponibilizado, sem a fragmentação da fitomassa, 96,7; 13,$6 ; 119,1 ; 31,2 ; 17,8 ; 14,1 ; 2.856$ e $19,1 \mathrm{~kg} \mathrm{ha}^{-1}$, e com a fragmentação 108,$6 ; 15,7 ; 107,7 ; 30,4 ; 19,9 ; 13,8 ; 2.826$ e 23,8 kg ha' ${ }^{-1}$, respectivamente, de N, P, K, Ca, Mg, S, C e Si.

Aos 91 DAM a fitomassa sem fragmentação tinha fornecido quantidades de nutrientes $\mathrm{N}, \mathrm{P}$ e K equivalentes a 220; 170 e $247 \mathrm{~kg} \mathrm{ha}^{-1}$, dos fertilizantes uréia, superfosfato simples e cloreto de potássio, respectivamente. Já a fitomassa que foi fragmentada as quantidades de N, P e K liberadas para o solo equivaliam a 247; 196 e $223 \mathrm{~kg} \mathrm{ha}^{-1}$ de uréia, superfosfato simples e 
Figura 2 - Teores de N (A), P (B), K (C), Ca (D), Mg (E), S (F), C (G) e Si (H) em g kg-1 de fitomassa do consórcio crotalária (Crotalaria juncea $)+$ milheto (Pennisetum glaucum) em função do tempo após o manejo (dias após manejo), sem ( -- ) e com (-Ð--) fragmentação mecânica. Botucatu, SP. Barras verticais são indicativas do valor de DMS a 5\% de probabilidade
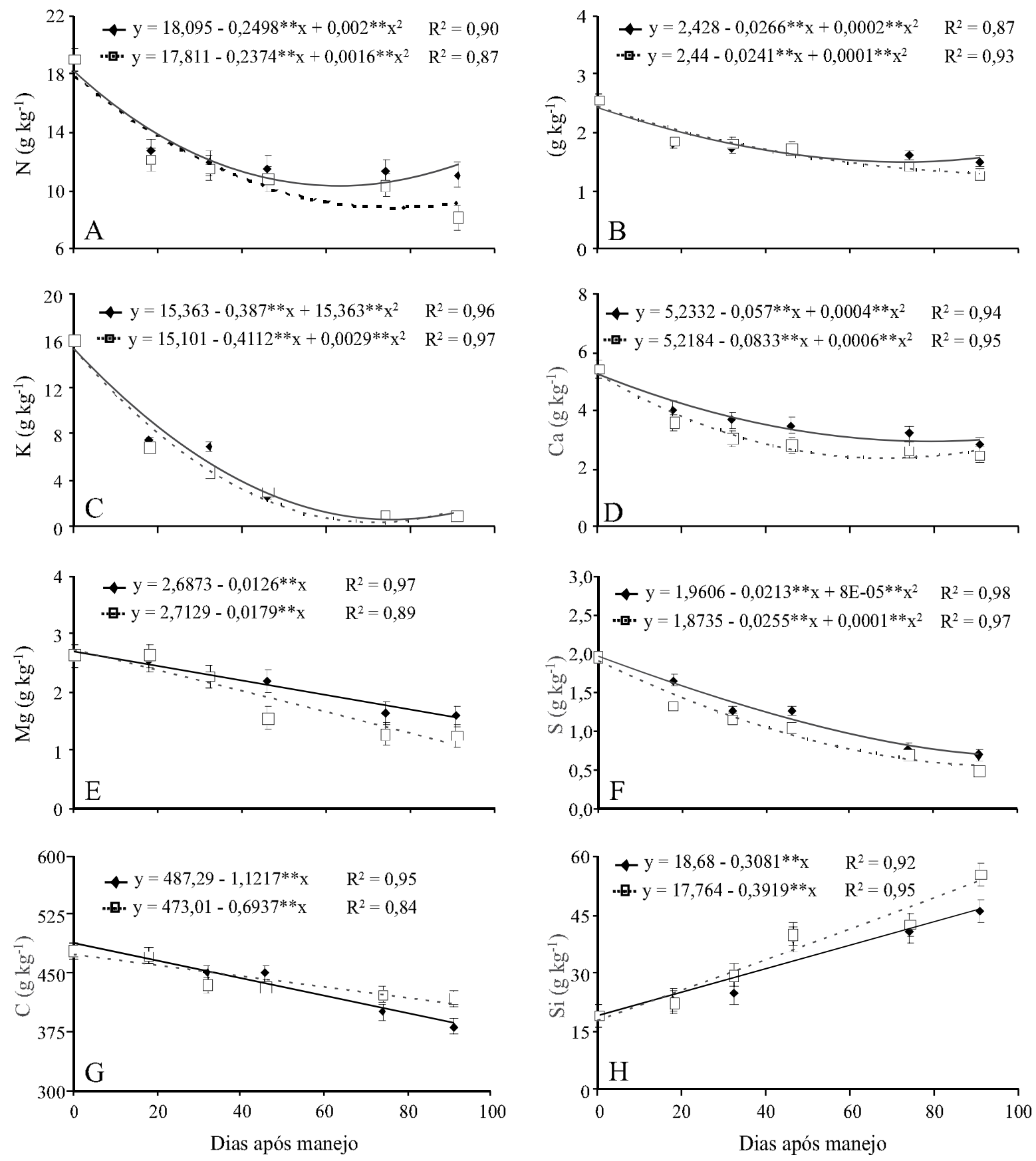

cloreto de potássio, respectivamente. Silva et al. (2010), observaram que a fitomassa de crotalária+milheto sem fragmentação proporcionou maior produtividade de grãos de mamona em sucessão, e atribuíram tal resultado à maior taxa de decomposição inicial da fitomassa quando fragmentada, liberando grandes

quantidades de nutrientes na fase de menor exigência da cultura. Assim, a fragmentação da fitomassa será vantajosa quando a fase de maior exigência nutricional da cultura em sucessão ocorrer próximo ao manejo.

Constata-se que, além da grande quantidade acumulada, foram liberadas porções significativas 
Figura 3 - Quantidade remanescente do N (A), P (B), K (C), Ca (D), Mg (E), S (F), C (G) e Si (H) em kg ha ${ }^{-1}$, na fitomassa do consórcio crotalária (Crotalaria juncea) + milheto (Pennisetum glaucum) em função do tempo após o manejo (dias após manejo), sem (-) e com ("---) fragmentação mecânica. Botucatu, SP. Barras verticais são indicativas do valor de DMS a $5 \%$ de probabilidade
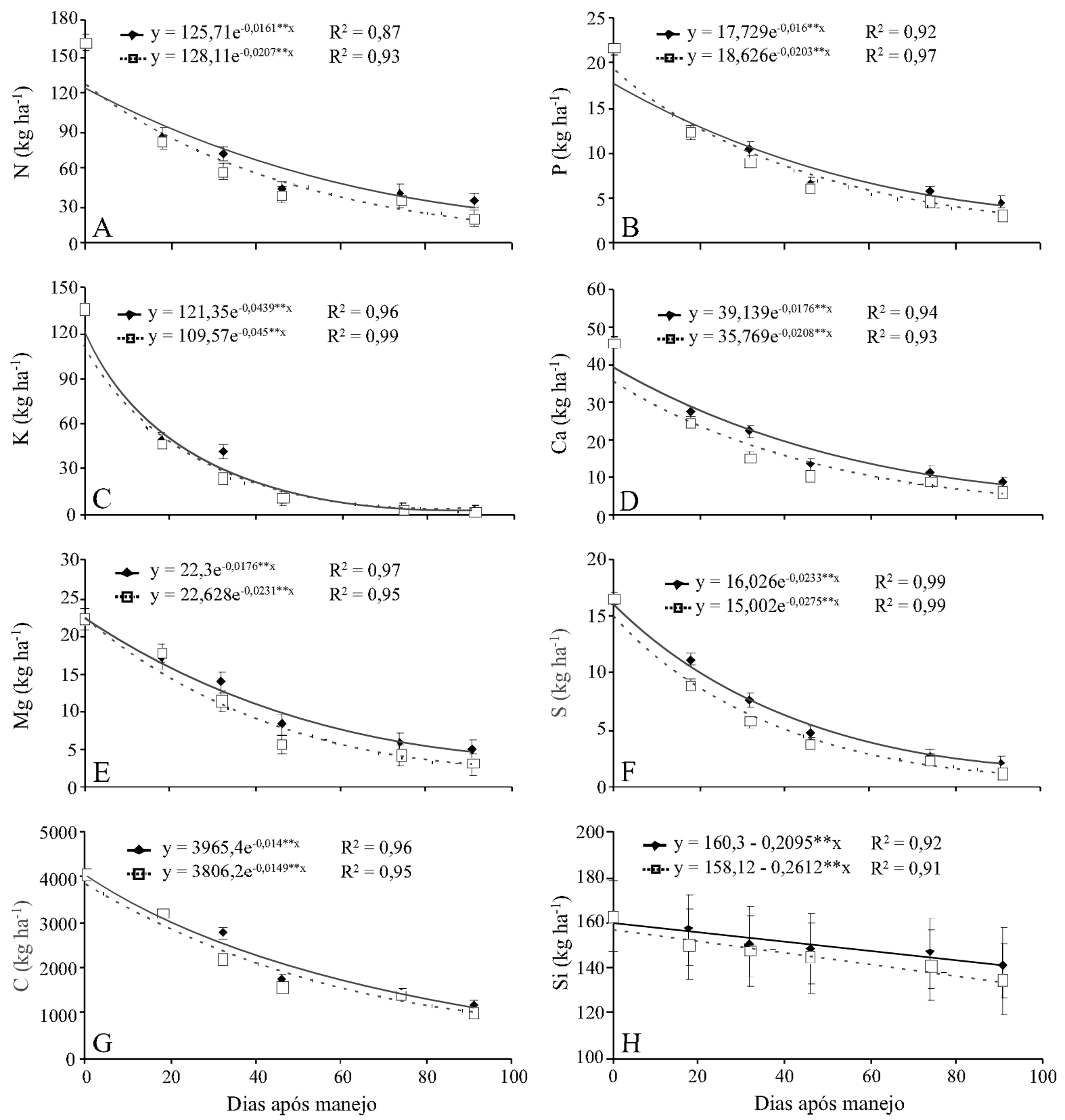

de nutrientes (Figura 4), o que poderia atender as necessidades da cultura sucedânea. Pois, da fitomassa com ausência de fragmentação mecânica, 50\% das quantidades totais de N, P, K, Ca, Mg, S e C acumuladas foram liberadas para o solo até $28 ; 31 ; 13 ; 30 ; 39$; 28 e 48 DAM, respectivamente. Já no manejo com fragmentação, as liberações de $50 \%$ das quantidades de

$\mathrm{N}, \mathrm{P}, \mathrm{K}, \mathrm{Ca}, \mathrm{Mg}, \mathrm{S}$ e C acumuladas na fitomassa foram aos $22 ; 27 ; 11 ; 21 ; 31 ; 22$ e 41 DAM, respectivamente. Assim, a reciclagem de nutrientes promovida pelo consórcio de plantas, independentemente do tipo de manejo adotado, minimizam os riscos de perdas por lixiviação, corroborando os resultados obtidos por Aita et al. (1994). 
Figura 4 - Porcentagem de N (A), P (B), K (C), Ca (D), Mg (E), S (F), C (G) e Si (H) (\%) remanescente na fitomassa do consórcio crotalária (Crotalaria juncea)+milheto (Pennisetum glaucum) em função do tempo após o manejo (Dias após manejo), sem ( $\bullet-\mathrm{e}$ com (- $\boxminus-\cdots)$ fragmentação mecânica. Botucatu, SP. Barras verticais são indicativas do valor de DMS a 5\% de probabilidade
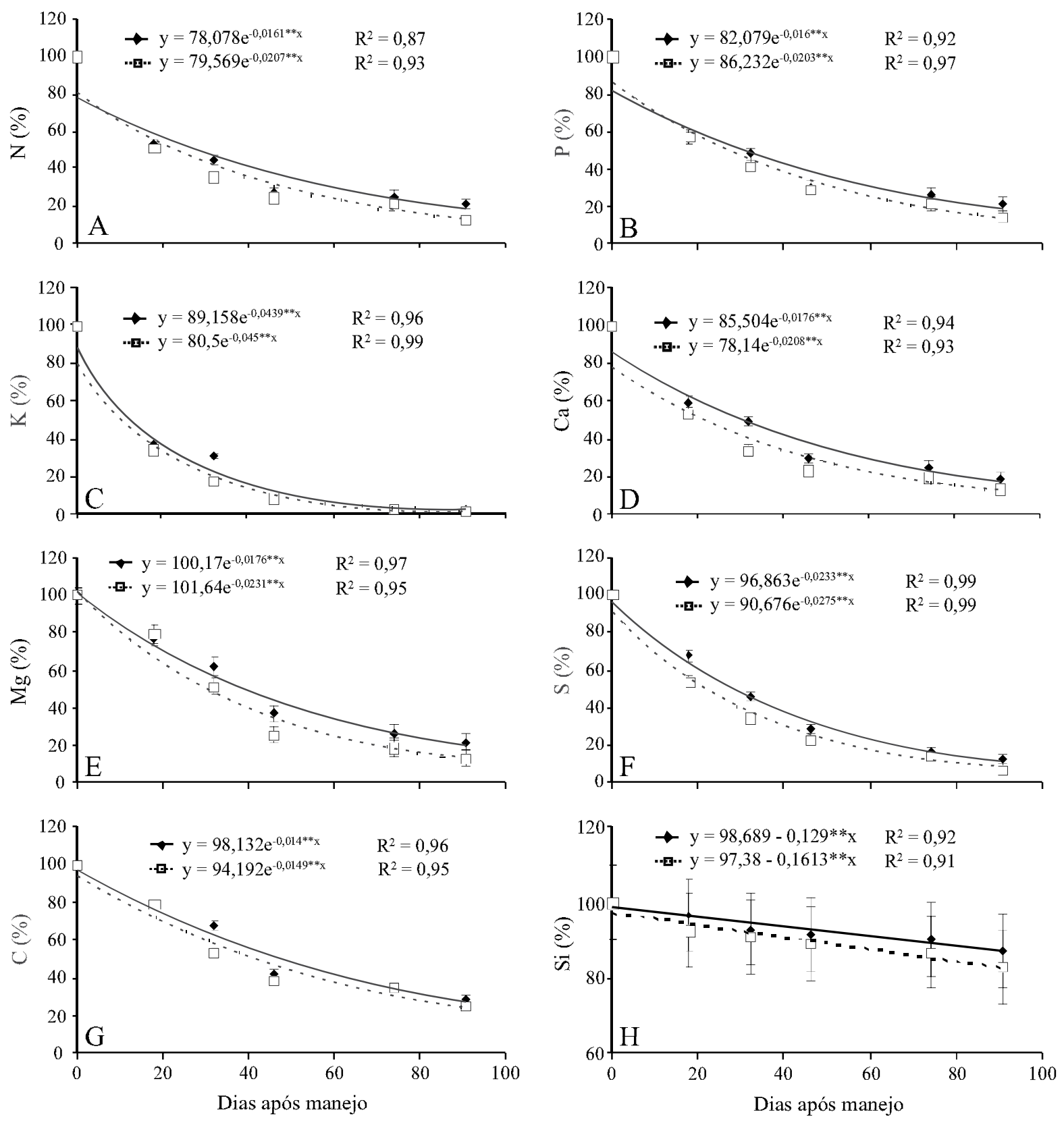

Quanto ao Si, observou-se acúmulo na fitomassa do consórcio, com liberação lenta e gradual, sendo que na última avaliação ainda restava na fitomassa 87 e $83 \%$ do Si acumulado, sem e com a fragmentação, respectivamente (Figuras $3 \mathrm{H}$ e $4 \mathrm{H}$ ).

Verificou-se que a maior velocidade de liberação dos elementos N, P, K, Ca, Mg, S e C ocorreu entre 0 e 18 DAM (Figuras 5A, 5B, 5C, 5D, 5E, 5F e 5G). Portanto,

a liberação desses elementos ocorre mais rapidamente na fase logo após o manejo, com redução continua, e posterior tendência à estabilização em valores próximos a zero. Crusciol et al. (2008) avaliando a taxa de liberação dos nutrientes da aveia preta, observaram maior taxa entre 10 e 20 DAM, para os nutrientes N, K, Ca e Mg.

No período entre 0 e $18 \mathrm{DAM}$, as taxas diárias de liberação dos elementos N, P, K, Ca, Mg, S e C 
Figura 5 - Taxa diária de liberação de N (A), P (B), K (C), Ca (D), Mg (E), S (F), C (G) e Si (H) (Kg ha ${ }^{-1}$ dia $\left.^{-1}\right)$ da fitomassa do consórcio crotalária (Crotalaria juncea) + milheto (Pennisetum glaucum) em função do tempo após o manejo (Dias após manejo),

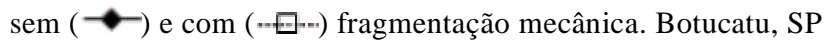
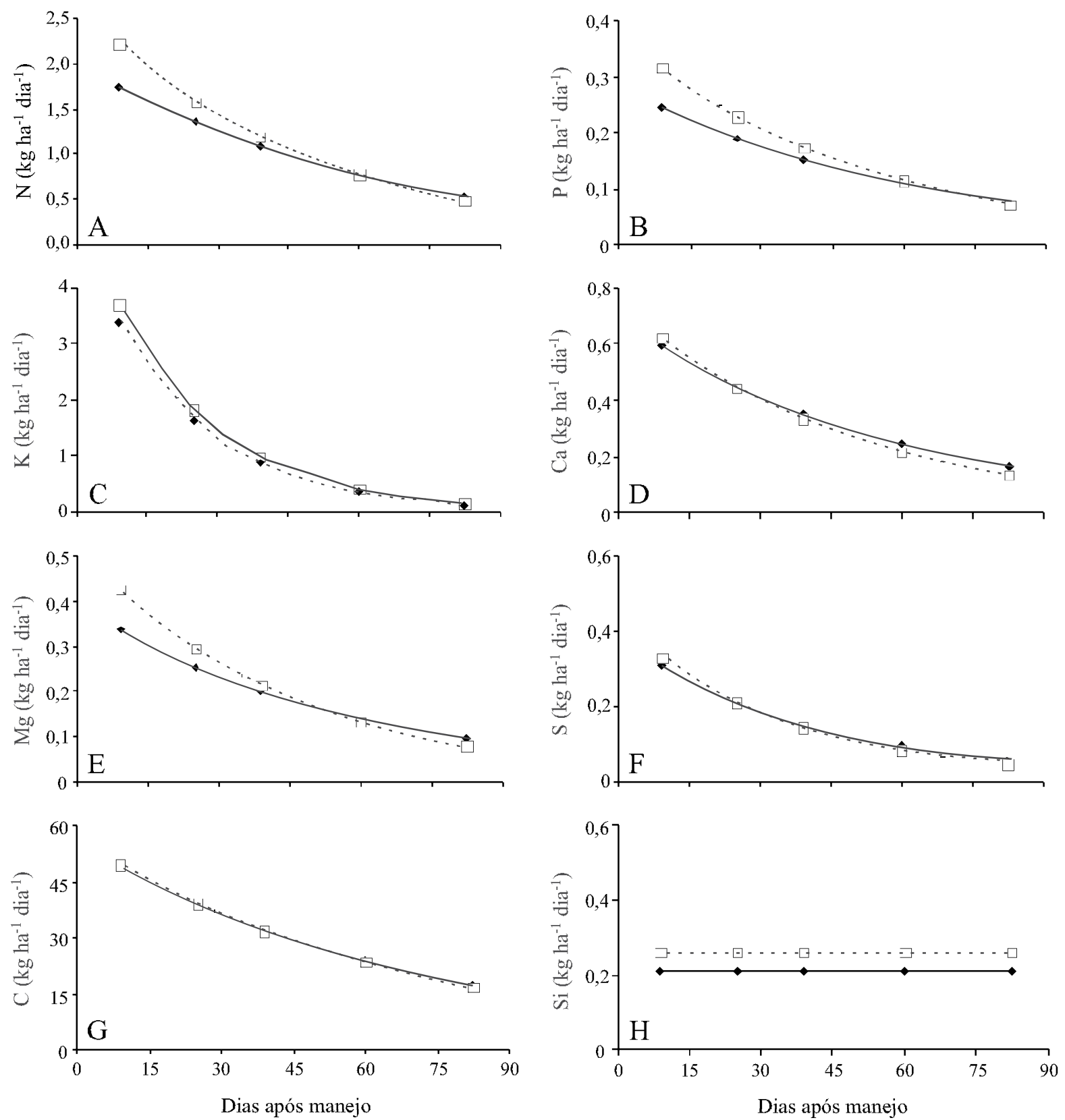

da fitomassa sem fragmentação atingiram intensidades de 1,$76 ; 0,25 ; 3,68 ; 0,59 ; 0,34 ; 0,30$ e $49 \mathrm{~kg} \mathrm{ha}^{-1} \mathrm{dia}^{-1}$, respectivamente. Já na presença da fragmentação mecânica as taxas foram de 2,$21 ; 0,32 ; 3,38 ; 0,62$; 0,$43 ; 0,33$ e $50 \mathrm{~kg} \mathrm{ha}^{-1} \mathrm{dia}^{-1}$, respectivamente, para $\mathrm{N}, \mathrm{P}, \mathrm{K}, \mathrm{Ca}, \mathrm{Mg}$, S e C (Figuras 5A; 5B; 5C; 5D; 5E; $5 \mathrm{~F}$ e $5 \mathrm{G})$. O Si teve liberação constante, com média de $0,24 \mathrm{~kg} \mathrm{ha}^{-1} \mathrm{dia}^{-1}$ durante todo o período avaliado,

nos dois manejos da fitomassa utilizados (Figura 5H). A fragmentação da fitomassa, realizada logo após o manejo químico, aumentou a liberação diária de $\mathrm{N}, \mathrm{P}$, K, Mg e Si (Figura 5).

A forma, a quantidade e a velocidade com que cada macroelemento foi liberado, independente do tipo de manejo da fitomassa, permitem inferir que, 
para maximizar o aproveitamento desses nutrientes, a implantação da cultura econômica deve ser realizada após o manejo da planta de cobertura.

\section{CONCLUSÕES}

1. A fragmentação da fitomassa do consórcio crotalária + milheto aumenta a taxa de decomposição e a liberação de N, P, Ca e S;

2. Com o passar do tempo ocorre aumento nas relações $\mathrm{C} / \mathrm{N}, \mathrm{C} / \mathrm{P}$ e $\mathrm{C} / \mathrm{S}$ e redução na relação $\mathrm{C} / \mathrm{Si}$ e na taxa de decomposição da fitomassa;

3. A máxima liberação diária de $\mathrm{N}, \mathrm{P}, \mathrm{K}, \mathrm{Ca}, \mathrm{Mg}, \mathrm{S}$ e $\mathrm{C}$ da palhada do consórcio crotalária + milheto ocorre no primeiro período de avaliação (0 a $18 \mathrm{DAM})$, e na última avaliação (91 DAM) pelo menos $80 \%$ de todos os macronutrientes $(\mathrm{N}, \mathrm{P}, \mathrm{K}, \mathrm{Ca}, \mathrm{Mg}$ e $\mathrm{S})$ já estão liberados ao solo;

4. O K é o nutriente mais rapidamente disponibilizado ao solo, restando aos 91 DAM, em média, apenas 1,5\% da quantidade total acumulada na palhada;

5. O Si é o elemento liberado mais lentamente ao solo, restando ainda aos 91 DAM, em média, $85 \%$ da quantidade total acumulada na palhada, isso porque a taxa de liberação de Si é baixa e constante, ocorrendo inclusive aumento do teor do elemento na fitomassa com o passar do tempo.

\section{AGRADECIMENTOS}

À FAPESP, pelo financiamento do projeto (Processo: 2004/10361-1), e ao CNPq, pela concessão de bolsa de Produtividade em Pesquisa para o segundo e terceiro autores.

\section{REFERÊNCIAS}

AITA, C. et al. Espécies de inverno como fonte de nitrogênio para o milho no sistema de cultivo mínimo e feijão em plantio direto. Revista Brasileira de Ciência do Solo, v. 18, n. 1, p. 101-108, 1994.

AITA, C.; GIACOMINI, S. J. Decomposição e liberação de nitrogênio dos resíduos culturais de plantas de cobertura de solo solteiras e consorciadas. Revista Brasileira de Ciência do Solo, v. 27, n. 4, p. 601-612, 2003.

BOER, C. A. et al. Biomassa, decomposição e cobertura do solo ocasionada por resíduos culturais de três espécies vegetais na região centro-oeste do Brasil. Revista Brasileira de Ciência do Solo, v. 32, n. 2, p. 843-851, 2008.
BOER, C. A. et al. Ciclagem de nutrientes por plantas de cobertura na entressafra em um solo de cerrado. Pesquisa Agropecuária Brasileira, v. 42, n. 9, p. 1269-1276, 2007.

CASTRO, O. M. et al. Perdas por erosão de nutrientes vegetais na sucessão soja/trigo em diferentes sistemas de manejo. Revista Brasileira de Ciência do Solo, v. 10, n. 3, p. 293-297, 1986.

CAZETTA, D. A.; FORNASIERI FILHO, D.; GIROTTO, F. Composição, produção de matéria seca e cobertura do solo em cultivo exclusivo e consorciado de milheto e crotalária. Acta Scientiarum. Agronomy, v. 27, n. 4, p. 575-580, 2005.

CRUSCIOL, C. A. C. et al. Persistência de palhada e liberação de nutrientes do nabo forrageiro no plantio direto. Pesquisa Agropecuária Brasileira, v. 40, n. 2, p. 161-168, 2005.

CRUSCIOL, C. A. C. et al. Taxas de decomposição e de liberação de macronutrientes da palhada de aveia preta em plantio direto. Bragantia, v. 67, n. 2, p. 481-489, 2008.

CRUSCIOL, C. A. C.; SORATTO, R. P. Nitrogen supply for cover crops and effects on peanut grown in succession under a no-till system. Agronomy Journal, v. 101, n. 1, p. 40-46, 2009.

CRUSCIOL, C. A. C.; SORATTO, R.P. Nutrição e produtividade do amendoim em sucessão ao cultivo de plantas de cobertura no sistema plantio direto. Pesquisa Agropecuária Brasileira, v. 42, n. 11, p. 1553-1560, 2007.

HERNANI, L. C.; KURIHARA, C. H.; SILVA, W. M. Sistema de manejo do solo e perdas de nutrientes e matéria orgânica por erosão. Revista Brasileira de Ciência do Solo, v. 23, n. 2, p. 145-154, 1999.

INOMOTO, M. M. et al. Avaliação em casa de vegetação do uso de sorgo, milheto e crotalária no manejo de Meloidogyne javanica. Tropical Plant Pathology, v. 33, n. 2, p. 125-129, 2008.

KHATOUNIAN, C. A. O manejo da fertilidade em sistemas de produção. In: CASTRO FILHO, C.; MUZILLIO, O. (Ed.). Uso e manejo dos solos de baixa aptidão agrícola. Londrina: IAPAR, 1999. p. 179-221. (Circular, 108)

KLIEMANN, H. J.; BRAZ, A. J. P. B.; SILVEIRA, P. M. Taxas de decomposição de resíduos de espécies de cobertura em latossolo vermelho distroférrico. Pesquisa Agropecuária Tropical, v. 36, n. 1, p. 21-28, 2006.

KORNDÖRFER, G. H. PEREIRA, H. S.; CAMARGO, M. S. Silicatos de cálcio e magnésio na agricultura. Uberlândia: GPSi - ICIAG - UFU, 2002. 23 p. (Boletim Técnico).

LEITE, L. F. C. et al. Decomposição e liberação de nutrientes de resíduos vegetais depositados sobre Latossolo Amarelo no Cerrado Maranhense. Revista Ciência Agronômica, v. 41, n. 1, p. 29-35, 2010.

MALAVOLTA, E.; VITTI, G. C.; OLIVEIRA, S. A. Avaliação do estado nutricional de plantas: princípios e aplicações. Piracicaba: Potafos, 1997. 308 p.

MARSCHNER, H. Mineral nutrition of higher plants. London: Academic Press, 1995. 889 p. 
MENEZES, L. A. S. et al. Produção de fitomassa de diferentes espécies, isoladas e consorciadas, com potencial de utilização para cobertura do solo. Bioscience Journal, v. 25 , n. 1 , p. 7-12, 2009.

MOREIRA, F. M. S.; SIQUEIRA, J. O. Microbiologia e bioquímica do solo. Lavras: UFLA, 2006. 729 p.

PAUL, E. A.; CLARK, F. E. Soil microbiology and biochemistry. San Diego: Academic Press, 1989. 275 p.

PERIN, A. et al. Produção de fitomassa, acúmulo de nutrientes e fixação biológica de nitrogênio por adubos verdes em cultivo isolado e consorciado. Pesquisa Agropecuária Brasileira, v. 39, n. 1, p. 35-40, 2004.

PERIN, A. et al. Sunnhemp and millet as green manure for tropical maize production. Scentia Agricola, v. 63, n. 5, p. 453459, 2006.

PERIN, A.; GUERRA, J. G. M.; TEIXEIRA, M. G. Cobertura do solo e acumulação de nutrientes pelo amendoim forrageiro. Pesquisa Agropecuária Brasileira, v. 38, n. 7, p. 791-796, 2003.

PINILLA, A. Estado actual de los estudios de fitolitos en suelos y planta. Madri: Centro de Ciências Medioambientais, 1997.
REIS, G. N. et al. Decomposição de culturas de cobertura no sistema plantio direto, manejadas mecânica e quimicamente. Engenharia Agrícola, v. 27, n. 1, p. 194-200, 2007.

Rosolem, C. A.; CAlONEGO, J. C.; FOLONI, J. S. S. Lixiviação de potássio da palhada de espécies de cobertura de solo de acordo com a quantidade de chuva aplicada. Revista Brasileira de Ciência do Solo, v. 27, n. 2, p. 355-362, 2003.

ROSOLEM, C. A.; PACE, L.; CRUSCIOL, C. A. C. Nitrogen management in maize cover crops rotations. Plant and Soil, v. 264, n. 1, p. 261-271, 2004.

SILVA, A. G. et al. Produção de fitomassa e acúmulo de nutrientes por plantas de cobertura e cultivo da mamona em sucessão no sistema plantio direto. Ciência Rural, v. 40, n. 10, p. 2092-2098, 2010.

TEDESCO, M. J.; WOLKWEISS, S. J.; BOHNEN, H. Análises de solo, plantas e outros materiais. Porto Alegre: Universidade Federal do Rio Grande do Sul, 1985. 188 p. (Boletim técnico, 5).

THOMAS, R. J.; ASAKAWA, N. M. Decomposition of leaf litter from tropical forage grasses and legumes. Soil Biology and Biochemistry, v. 25, n. 10, p. 1351-1361, 1993. 